Progress in Flight Physics 5 (2013) 23-42

DOI: $10.1051 /$ eucass/201305023

(C) Owned by the authors, published by EDP Sciences, 2013

\title{
FINE-TUNING OF DDES AND IDDES FORMULATIONS TO THE $k-\omega$ SHEAR STRESS TRANSPORT MODEL
}

\author{
M. S. Gritskevich ${ }^{1}$, A. V. Garbaruk ${ }^{1}$, and F. R. Menter $^{2}$ \\ ${ }^{1}$ St. Petersburg State Polytechnical University \\ St. Petersburg 195251, Russia \\ ${ }^{2}$ Software Development Department, ANSYS \\ Otterfing 83714, Germany
}

\begin{abstract}
Modifications are proposed of two recently developed hybrid CFD (computational fluid dynamics) strategies, Delayed Detached Eddy Simulation (DDES), and DDES with Improved wall-modeling capability (IDDES). The modifications are aimed at fine-tuning of these approaches to the $k-\omega$ SST (shear stress transport) background RANS (Reynolds-averaged Navier-Stokes) model. The first one includes recalibrated empirical constants in the shielding function of the SpalartAllmaras (SA) based DDES which are shown to be suboptimal (not providing a needed level of elimination of the Model Stress Depletion (MSD)) for the SST-based DDES model. For the SST-IDDES variant, in addition to that, a simplification of the original SA-based formulation is proposed, which does not cause any visible degradation of the model performance. Both modifications are extensively tested on a range of attached and separated flows (developed channel, backward-facing step, periodic hills, wall-mounted hump, and hydrofoil with trailing edge separation).
\end{abstract}

\section{INTRODUCTION}

Industrial CFD simulations increasingly rely on Scale-Resolving Simulation (SRS) models, which resolve at least a part of the turbulence spectrum in at least a part of the flow domain. Due to the excessive costs of classical Large Eddy Simulation (LES) for high-Reynolds number industrial simulations, hybrid and/or zonal RANS-LES models are quickly becoming the models of choice for such applications. A result of the intensive research in this area, a significant number of models have been proposed in recent years [1] making a comparison

This is an Open Access article distributed under the terms of the Creative Commons Attribution License 2.0, which permits unrestricted use, distribution, and reproduction in any medium, provided the original work is properly cited. 
and selection of the most appropriate model a daunting task. However, only a small number of model formulations are used in today's industrial CFD codes and can roughly be categorized in the following way:

- Improved Unsteady RANS (URANS) models which allow the formation of resolved turbulent structures in unstable flows without an explicit impact of the grid spacing on the RANS model formulation. The most widely used model of this type is the Scale-Adaptive Simulation (SAS) variant. These models are relatively safe to use, as they provide a (U)RANS fallback position for underresolved grids and/or time steps. On the downside, such models require relatively strong flow instabilities in order to switch to SRS mode;

- Detached Eddy Simulation (DES) models, which switch explicitly between RANS and LES model formulations based on the local grid spacing and turbulent length scale. The original intent of DES was to be run in RANS mode for attached boundary layers and to switch to LES mode in large separated (detached) flow regions. The explicit switch to the LES model is, however, not accompanied by a corresponding transfer of modeled (RANS) turbulence to resolved (LES) turbulence. As with SAS, DES relies on inherent flow instability for a quick generation of such resolved content. Due to the direct impact of the grid spacing on the RANS model, DES models require more carefully crafted grids to avoid inappropriate behavior. On the other hand, DES models allow a local reduction in eddy-viscosity by grid refinement in the 'switch' region between RANS and LES, which, in turn, can help in the formation of unsteady content, for flows where models like SAS would remain in (U)RANS mode;

- Wall Modeled LES (WMLES) models, which aim at reducing the strong Reynolds number dependency of classical LES for wall-bounded flows. This is typically achieved by covering only the inner-most part of the boundary layer in RANS mode and resolving most of the turbulence inside the boundary layer by LES techniques. This avoids the need of resolving the smallest and most Reynolds number dependent turbulent eddies above the viscous sublayer. As the turbulent eddies inside the attached boundary layer are typically still much smaller than 'detached' eddies, WMLES requires a substantially higher computational effort than classical DES; and

- Zonal (or embedded) LES models, in which the user divides the domain into separate regions where RANS and LES models are applied respectively. At the interface between an upstream RANS and a downstream LES region, synthetic turbulence is typically inserted into the simulation, providing a clear transfer of turbulence energy from the modeled to resolved content. Obviously, zonal formulations can be combined with the use of a WMLES formulation in the 'LES' zone. 
The current paper will focus on different aspects and variants of the DES model formulation. While the original DES model is straightforward and simple, DES is, nevertheless, one of the most difficult models to use in complex applications. The user requires not only a basic understanding of the model behavior, but also has to follow relatively intricate grid generation guidelines to avoid undefined simulation behavior somewhere between RANS and LES. In addition, several variants of the DES model, like DDES and IDDES have been proposed with rather different characteristics, making model selection and interpretation of results challenging.

Problematic behavior of standard DES has been reported by Menter et al. [2] who demonstrated that an artificial separation could be produced for an airfoil simulation when refining the max cell edge length $\left(\Delta_{\max }\right)$ inside the wall boundary layer below a critical value of $\Delta_{\max } / \delta<0.5 \sim 1$ where $\delta$ is the local boundary layer thickness. This effect was termed Grid Induced Separation (GIS) as the separation depends on the grid spacing and not on the flow physics. Grid induced separation is obviously produced by the effect of a sudden grid refinement which changes the DES model from RANS to LES, without balancing the reduction in eddy-viscosity by resolved turbulence content. Spalart [3] coined the term MSD which refers generally to the effect of reduction of eddy-viscosity from RANS to LES without a corresponding balance by resolved turbulent content. In other words, GIS is a result of MSD. Modeled stress depletion is essentially a result of insufficient flow instability near the switch between RANS and LES model formulation. For that reason, the switch from the RANS to the LES model inside wall boundary layers is not desirable. Grid induced separation can, in principle, be avoided by shielding the RANS model from the DES formulation for wall boundary layers. This was proposed by Menter et al., who used the blending functions of the SST model for that purpose [2]. Later, Spalart et al. [3] proposed a more generic formulation of the shielding function, which depends only on the eddy-viscosity and the wall distance. It can, therefore, in principle, be applied to any eddy-viscosity based DES model. The resulting formulation was termed DDES [3]. While the shielding function developed in [3] was considered generic, it was essentially calibrated for the SA one-equation RANS model.

It will be shown that a recalibration is required if the same function is to be applied to other models like the SST two-equation model used in the current work. It is important to emphasize that the development and/or calibration of DDES shielding functions requires a delicate balance between the need of shielding the boundary layer and the desire of not inhibiting the formation of turbulent structures in the 'switch' zone between attached (RANS) and detached (LES) flow. Overly conservative shielding would allow a high degree of mesh refinement inside the boundary layer without any impact on the RANS model, but would suppress the formation of resolved turbulence in detached flow regions not sufficiently removed from the walls (e. g., backstep flows, tip gap flows in axial turbines, etc.). 
Another interesting aspect spurring many discussions and model enhancements resulted from the application of the original DES model as a WMLES formulation. Obviously, this was not the original intent of the model, and it resulted in a relatively strong Logarithmic Layer Mismatch (LLM) between the inner RANS and the outer LES regions. Nevertheless, these tests indicated that DES could be developed into a suitable WMLES formulation, resulting in the formulation of the IDDES model [4]. The IDDES model features several rather intricate blending and shielding functions, which allow using this model both in DDES and WMLES mode. These functions will be revisited, again in combination with the SST model, and some recalibration and simplifications will be proposed, in an attempt of making the model both simpler and more reliable.

\section{BRIEF DESCRIPTION OF THE NUMERICS}

All the simulations in the present study have been carried out with the use of the ANSYS-Fluent 13 CFD code [5]. Within this code, the governing equations are written in a transient formulation for the DDES and IDDES and in a steady-state formulation for all the steady RANS computations. For all the considered flows, the incompressible fluid assumption was utilized. The numerical method of the code uses a finite volume method on unstructured grids with a cell-centered data arrangement.

The equations are solved with the use of implicit point Gauss-Seidel method with a Rhie-Chow flux correction [6] which is aimed at suppressing unphysical pressure oscillations. An algebraic multigrid approach is applied for convergence acceleration by computing corrections on a series of grids. For the RANS computations, the coupled steady-state solver [5] is employed, whereas for DDES and IDDES, a noniterative time advancement procedure $[5,7,8]$ is used which allows integrating the governing equations in time without inner iterations on each time step.

The inviscid fluxes are approximated with the use of the second-order upwind scheme [5] for RANS and with the second-order centered scheme [5] for DDES and IDDES. The time derivatives in the latter simulations are approximated with the use of the three-layer second-order backward Euler scheme.

\section{TEST CASES DESCRIPTION}

\subsection{Developed Channel}

Simulations of this flow were carried out at the Reynolds numbers based on friction velocity $u_{\tau}$ and channel height $H$ equal to 395,2400 , and 18,000 . The flow was driven with a constant pressure gradient $d p / d x=-2 \rho u_{\tau} / H$ where $p$ is 
the pressure and $\rho$ is the density. This pressure gradient was taken into account in the governing equation via a source term in the momentum equations and periodic boundary conditions were imposed not only in the spanwise direction $z$, but also in the streamwise direction $x$. Note that within such an approach, the bulk velocity of the flow is not specified and should be obtained as a part of the solution, which means that it could be different with different turbulence models. The computational domain used in the present study was also the same as that used in [4], namely, its size was equal to $4 H$ in the streamwise direction and $1.5 \mathrm{H}$ in the spanwise direction. For all the considered Reynolds numbers, the computational grid was the same with grid-step in streamwise and spanwise directions equal to $0.05 H$ and $0.025 H$, respectively. In the wall normal direction, different grids were used providing a sufficient resolution $\left(\Delta y_{w}^{+}<1\right.$ near the wall) at different Reynolds numbers. A nondimensional time step was $\Delta t=0.02$ which ensured the Courant-Friedrichs-Lewy (CFL) number to be less than 1 in the entire domain.

\subsection{Backward-Facing Step}

This flow has been experimentally studied in the work of Vogel and Eaton [8] with a Reynolds number based on a bulk velocity and on the step height $H$ equal
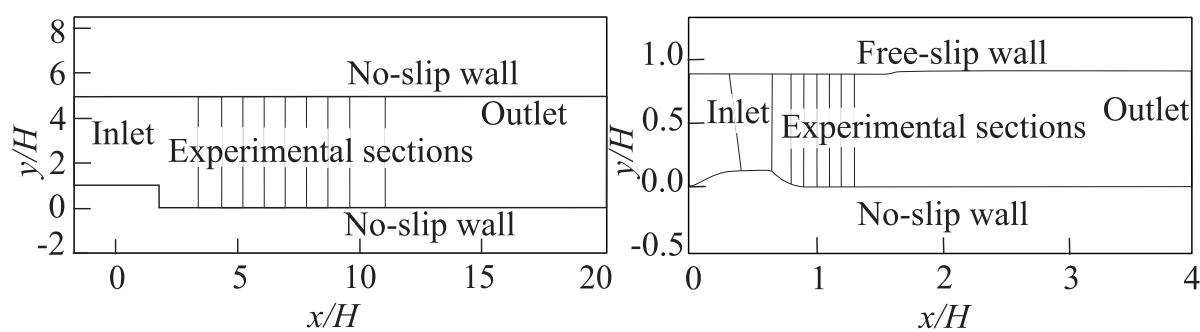

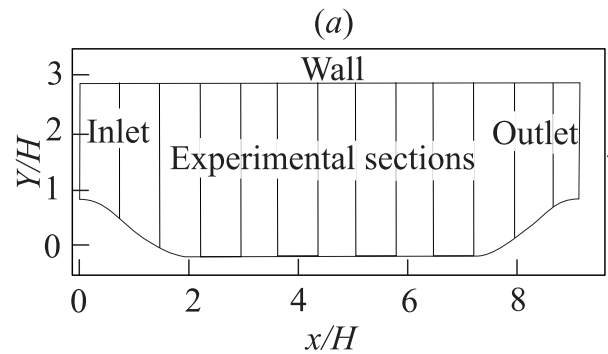

(b)

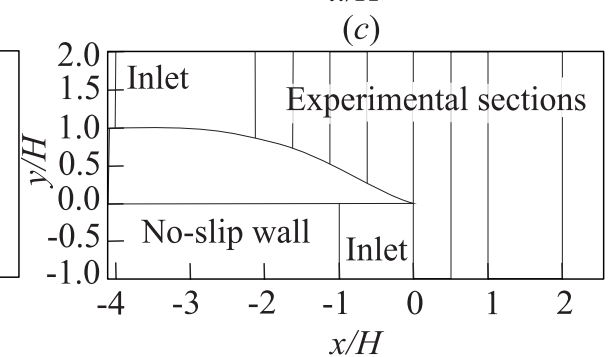

(d)

Figure 1 Computational domain with experimental sections for backward facing step $(a)$, periodic hills $(b), 2 \mathrm{D}$ hump $(c)$, and hydrofoil with trailing edge separation $(d)$ 


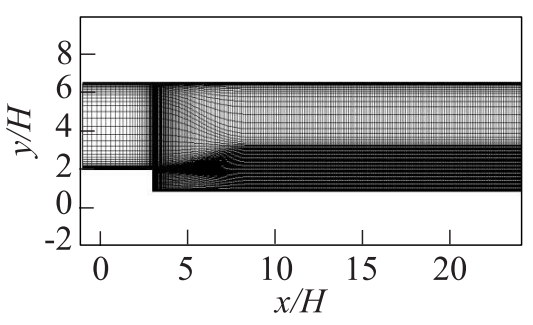

(a)

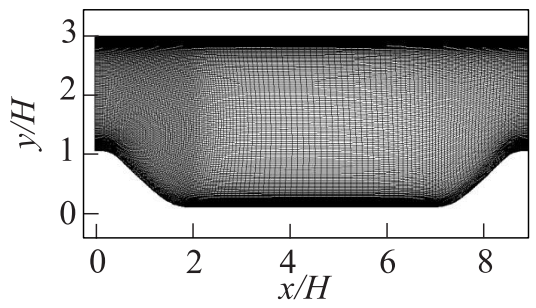

(b)

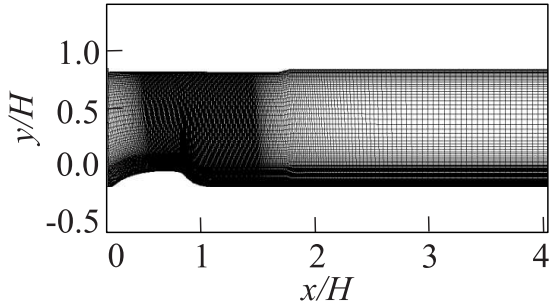

(c)

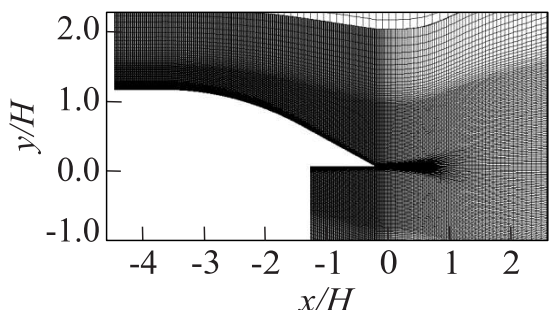

(d)

Figure 2 Computational domain with experimental sections for backward facing step $(a)$, periodic hills $(b), 2 \mathrm{D}$ hump $(c)$, and hydrofoil with trailing edge separation $(d)$

to 28,000 . The height of the channel upstream of the step was equal to $4 H$. Following previous simulations of this flow with the use of SA-based DES and DDES models $[3,6,9]$, the computational domain (Fig. 1a) in the present study extended from $-3.8 \mathrm{H}$ to $20 \mathrm{H}$ in the streamwise direction $(x=0$ corresponds to the step location). In the spanwise direction, the size of the domain was $4 H$.

The computational grid used in the simulation had 2.25 million hexahedral cells (2.3 million nodes) providing a near-wall resolution in wall units of less than one (Fig. $2 a$ ). The maximum grid-step in streamwise and spanwise directions was equal to $0.1 H$ and $0.05 H$, respectively. A nondimensional time step of $\Delta t=0.02$ ensured the CFL number of less than 1 in the entire domain. At the inlet condition, steady-state RANS profiles were imposed with unsteadiness resulting from the inherent flow instability past the step.

\subsection{Flow Over Periodic Two-Dimensional Hills}

This flow is a popular test case for validation of turbulence models with separation and reattachment. It served as the test case of two ERCOFTAC SIG15 Workshops $[10,11]$ and is included in the ERCOFTAC database (case 81) where details of the geometry are given. In the present simulations, the Reynolds number based on the hill height, $H$, and the bulk velocity, $U_{b}$, was equal to 10600 . 
Following Breuer et al. LES [12], the length of the computational domain was equal to $9 H$ and its size in the spanwise direction was $4.5 H$ (see Fig. $1 b$ ). The computational grid contained about 1.5 million hexahedral cells which correspond to $161 \times 161 \times 61$ nodes in the $x, y$, and $z$ directions, respectively (see Fig. $2 b$ ). The maximum grid-step in streamwise and spanwise directions was equal to $0.12 H$ and $0.075 H$, respectively. The grid ensured the values of $\Delta y_{w}^{+}$ to be less than 1 for both the hill-wall and upper straight wall. The nondimensional time step in the simulations was $\Delta t=0.02$ which corresponds to the CFL number of less than 1 in the entire domain. On the upper and lower walls of the channel no slip-conditions were applied, whereas the boundary conditions in the spanwise and streamwise directions were set to periodic.

\subsection{Wall-Mounted Two-Dimensional Hump Flow}

This flow has been studied experimentally by Greenblatt et al. [10] and, similar to the flow over the periodic hills, it has been used as a benchmark in a number of CFD studies $[13,14]$. The present simulations were conducted with a Reynolds number of $9.36 \cdot 10^{5}$ based on the free-stream velocity $U_{\infty}$ and hump chord $C$. The simulation of this flow was performed in two stages:

(1) two-dimensional (2D) RANS computation has been carried out in the full domain extending from $-2.14 C$ to $4 C$ ( 0 corresponds to the hump's beginning) with a grid of $4.0 \cdot 10^{4}$ hexahedral cells. The inflow boundary conditions for RANS were imposed based on the preliminary flat plate boundary layer computations up to the flow section $x / C=-2.14\left(\operatorname{Re}_{\theta}=7200\right)$ where the flow parameters were measured in the experiment. Other than that, the upper (straight) wall of the channel, where the free-slip wall conditions are specified, was slightly constricted to reproduce a blockage effect of the end plates in the experimental configuration [15]; and

(2) IDDES stage of the simulation where the computational domain (see Fig. $1 c$ ) extends from $0.4 C$ to $4 C$ (its inlet section is placed on the hump plateau), and its size in the spanwise direction is equal to $0.2 C$. The inflow boundary conditions are based on the RANS solution at $x / C=0.4$ known from the previous simulation, whereas the inflow turbulent content needed for activating the WMLES branch of the IDDES model is created with the use of the recently proposed synthetic turbulence generator [11]. In the spanwise direction, periodic boundary conditions are imposed.

The computational grid in the IDDES simulation has about 1.6 million hexahedral cells with maximum grid-step in streamwise and spanwise directions equal to $0.008 C$ and $0.004 C$, respectively (see Fig. $2 c$ ). The nondimensional time step in the simulation is $\Delta t=0.001$ which leads to a CFL number less than 1 in the entire domain. 


\subsection{Hydrofoil with a Trailing Edge Separation}

This flow investigated in the experiments of Blake [13] is characterized by a shallow separation bubble with unfixed separation point and presents a challenging test to CFD. The Reynolds number based on the free stream velocity and the hydrofoil chord is equal to $2.2 \cdot 10^{6}$ or $1.01 \cdot 10^{5}$ based on its thickness $H$.

Similar to the $2 \mathrm{D}$ hump considered in the previous subsection, the simulation of the flow is performed with the use of the two-stage, RANS-IDDES, approach. The 2D RANS computation is carried out for the entire hydrofoil; the computational domain extends from $x / H=-60$ to $x / H=20$ in $x$ direction $(x=0$ corresponds to the hydrofoil trailing edge) and from $y / H=-40$ to 40 in the $y$ direction (see Fig. $1 d$ ). The RANS grid has $1.2 \cdot 10^{5}$ hexahedral cells.

The IDDES domain starts at $x / H=-4$ under the hydrofoil and at $x / H$ $=-1$ above it and extends up to $x / H=20$ in the wake and its size in the spanwise direction is equal to $0.5 H$. The inflow boundary conditions for IDDES are based on the RANS solutions at $x / H=-4$ under and at $x / H=-1$ above the hydrofoil and the inflow turbulent content is again created with the use of the synthetic turbulence generator [11]. In the spanwise direction, periodic boundary conditions are imposed. The IDDES grid has about 3.5 million hexahedral cells with 50 cells in spanwise direction. The grid-steps in the streamwise and spanwise directions are equal to $0.01 H$ and $0.01 H$, respectively (see Fig. $2 d$ ), and the grid in the wall-normal direction is designed so that the near-wall $\Delta y_{w}^{+}$is less than 1 in the entire domain. The nondimensional time step is $\Delta t=0.005$ which corresponds to the CFL number less than 1 in the entire domain.

\section{RECALIBRATION OF THE ORIGINAL DDES CONSTANTS TO THE $k-\omega$ SHEAR STRESS TRANSPORT MODEL}

The original SST-DDES formulation combines the SST-DES formulation of Travin et al. [14] with the DDES shielding functions of Spalart et al. [3]. The original SST-DES model starts to decrease the eddy viscosity for $h_{\max } / \delta<0.8$ and the purpose of the empirical shielding function is to preserve the eddy viscosity from degradation up to $h_{\max } / \delta=0.1$ (in fact even less). The empirical delay function $f_{d}$ involved in the DDES approach reads as follows [3]:

$$
f_{d}=1-\tanh \left[\left(C_{d 1} r_{d}\right)^{C_{d 2}}\right] ; \quad r_{d}=\frac{\nu_{t}+\nu}{\kappa^{2} d_{w}^{2} \sqrt{0.5\left(S^{2}+\Omega^{2}\right)}} .
$$

Here, $\nu_{t}$ and $\nu$ are the eddy and molecular viscosities, respectively; $S$ and $\Omega$ are the strain rate and vorticity tensor invariants; $\kappa=0.41$ is the von Karman constant; and $d_{w}$ is the distance to the wall. 


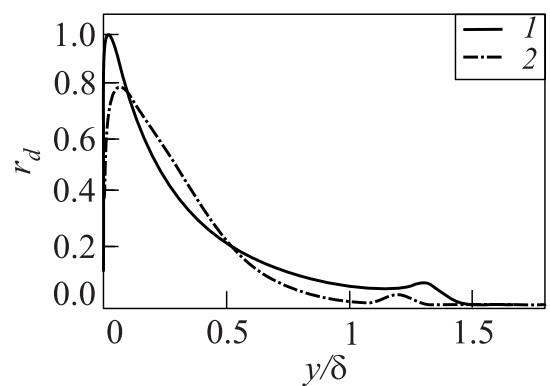

(a)

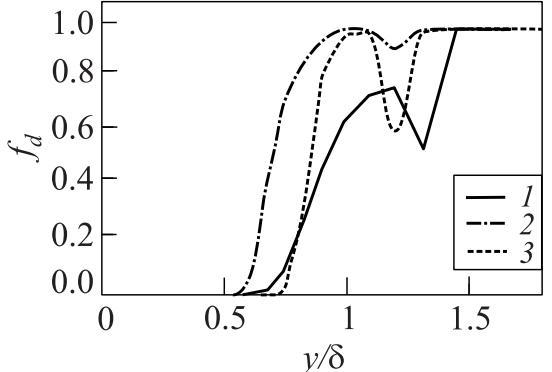

(b)

Figure 3 Comparison between SA- (1) and SST-based DDES $\left(2-C_{d 1}=8\right.$; and $\left.3-C_{d 1}=20\right)$ for flat plate boundary layer: $(a) r_{d}$ quantity, and $(b) f_{d}$ shielding function

Based on the computations of a zero-pressure-gradient boundary layer with the use of the SA RANS model and SA-based DDES carried out in [3] on a fairly ambiguous grid (with a target value of the grid-spacing equal to one-tenth of the boundary layer thickness), the values of the constants $C_{d 1}$ and $C_{d 2}$ involved in the quantity $r_{d}$ have been set equal to 8 and 3 , respectively. However, as shown in Fig. $3 a$, the profiles of $r_{d}$ are different for the SA-DDES and SSTDDES models when using the same shielding function. Thus, with these values of the constants, the SST-DDES delay function turns out to be equal to 1 in a significantly narrower domain than the SA-DDES function, which results in a less-reliable shielding of the boundary layer for the SST-based DDES compared to the SA-based DDES (see Fig. $3 b$ ).

A series of SST-based DDES computations with different values of the constants has shown that in order to ensure nearly the same protection of the SSTbased DDES model from a premature switching to LES mode as for the SA-based DDES model, the value of $C_{d 1}$ should be set equal to 20 , whereas the constant $C_{d 2}$ should be kept the same as in the SA-based DDES (see Fig. $3 b$ ).

A significant improvement of the SST-based DDES performance on ambiguous grids ensured by the use of the new set of the constants is illustrated by Fig. 4 . Figure 4 presents the results of the SST-DDES model for the flat plate boundary layer computed in RANS mode with the DDES option activated. In this simulation, the maximum grid-spacing $h_{\max }$ involved in the DDES formulation was abruptly changed from $\delta$ (boundary layer thickness) to $0.1 \delta$ at $\operatorname{Re}_{x}=5 \cdot 10^{6}$. This situation may well be the case in complex flows, e.g., in the vicinity of geometry singularity. As seen in Fig. $4 a$ for the SST-based DDES model with the "standard" (recommended for the SA-based DDES) value of the $C_{d 1}$ constant equal to 8 , the shielding of the model from the premature switching inside the boundary layer to the LES mode typical for the original DES is not completely 


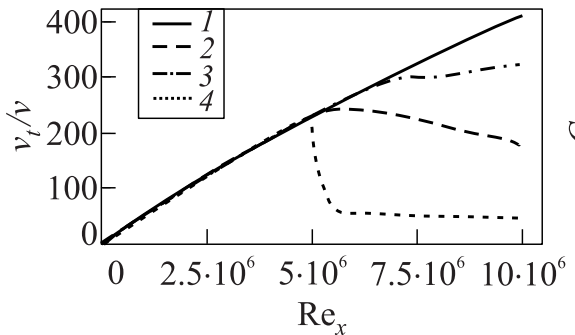

(a)

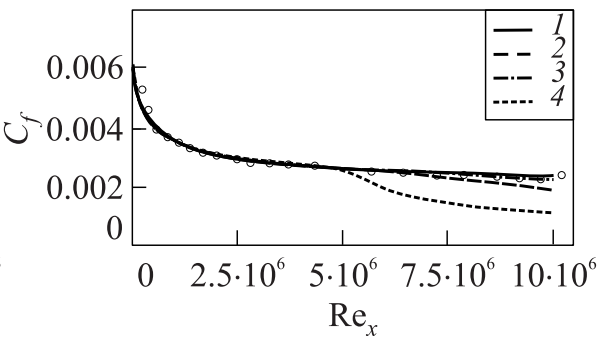

(b)

Figure 4 Effect of constant $C_{d 1}$ on the flat plate flow predicted by SST-based DDES: (a) distribution of eddy viscosity maximum value in each profile over the plate, and (b) distribution of skin friction coefficient distribution over the plate; 1 - SST; 2 DDES, $C_{d 1}=8 ; 3-$ DDES, $C_{d 1}=20 ; 4-$ DES; and signs - experiment

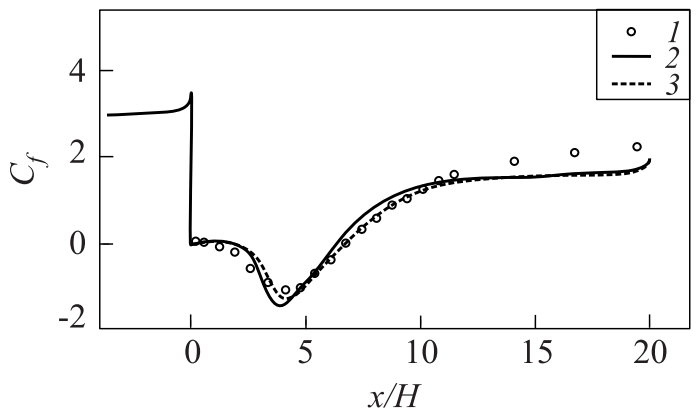

(a)

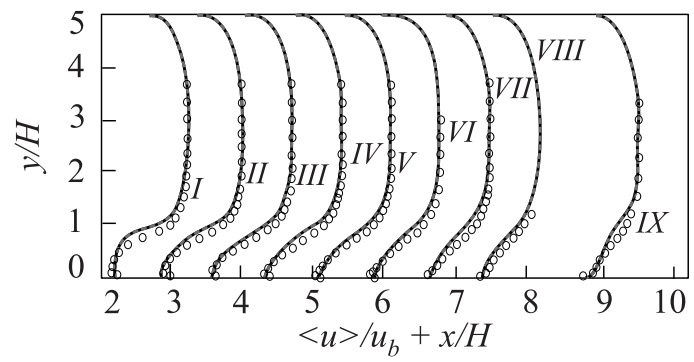

(b)

Figure 5 Effect of constant $C_{d 1}$ on the BFS mean flow predicted by SST-based DDES: (a) skin friction coefficient distribution over the step-wall and $(b)$ profiles of streamwise velocity $\langle u\rangle$. Profiles are plotted at $x / H=2.2(I), 3.0(I I), 3.7(I I I), 4.5(I V), 5.2(V)$, $5.9(V I), 6.7(V I I), 7.4(V I I I)$, and $8.7(I X): 1$ - experiment; $2-\mathrm{DDES}, C_{d 1}=8$; and $3-$ DDES, $C_{d 1}=20$ 
eliminated. A significant drop of the maximum eddy viscosity per profile compared to the SST RANS eddy viscosity is observed (see Fig. 4a). This naturally leads to a tangible deviation of the friction coefficient from the SST RANS curve (Fig. $4 b$ ) and, more importantly, could result in GIS under adverse pressure gradient conditions. In contrast to this, with $C_{d 1}=20$, both eddy viscosity and skin friction predicted by the SST DDES are virtually the same as those computed with the SST RANS model, which means that the MSD does not take place even on the considered very fine grid. Even with the new limiter, the RANS mode for boundary layer computations will be affected once $\Delta_{\max } / \delta<0.1$. This can be seen to happen for the current grid at $\operatorname{Re}_{x} \sim 7 \cdot 10^{6}$ (see Fig. $4 a$ ).

Overly conservative shielding of the DDES model can, in principle, result in impairing the turbulence resolving capability of the DDES model in separated flow regions. In order to make sure that this does not occur with $C_{d 1}=20$, the backward-facing step flow (see subsection 3.2) has been computed.

A comparison of the mean flow characteristics predicted by the two simulations with each other and with the experimental data [8] is presented in Fig. 5. As seen in the figure, the difference between the friction distributions over the step-wall and velocity fields computed with the different values of the constant is marginal, and both solutions agree well with the data. Thus, the increase of the $C_{d 1}$ constant from 8 to 20 does not cause any noticeable degradation of the SST-based DDES model in LES mode and can be considered as both robust (ensuring a sufficient shielding of SST-DDES from MSD in the attached flow regions) and safe (not leading to a degradation of turbulence resolving capabilities of the model) in the separation regions.

\section{OPTIMIZATION OF THE IDDES MODEL FORMULATION FOR THE SHEAR STRESS TRANSPORT MODEL}

The IDDES approach [4] presents a combination of DDES with another hybrid model aimed at WMLES. In this combined approach, the empiric function providing shielding of the DDES branch of the model from MSD is similar to the function $f_{d}$ in DDES and reads as follows [4]:

$$
f_{d t}=1.0-\tanh \left[\left(C_{d t 1} r_{d t}\right)^{C_{d t 1}}\right] .
$$

Here, the values of the constants $C_{d t 1}$ and $C_{d t 2}$ are the same as those in the SA-DDES, i. e., 8 and 3, respectively [4]. Thus, taking into account the results presented in section 3 , for the SST-based IDDES, the value of $C_{d t 1}$ constant should also be set to 20 . In order to ensure that this does not damage the wallmodeling capability of the IDDES branch, simulations have been carried out 
for the developed flow in a plane channel, where the wall-modeling capability is essential for computing flows at high Reynolds numbers. In order to also test the model in a flow where both of its branches (DDES and WMLES) are active, the simulation of the Bozzolo-Ferrante-Smith (BFS) presented in the previous section was repeated with the use of the SST-based IDDES.

As a first test, the periodic channel flow was calculated (see subsection 3.1). The results of the simulations obtained with the use of the SST-IDDES model with two values of the constant $C_{d t 1}$ and their comparison with the empirical
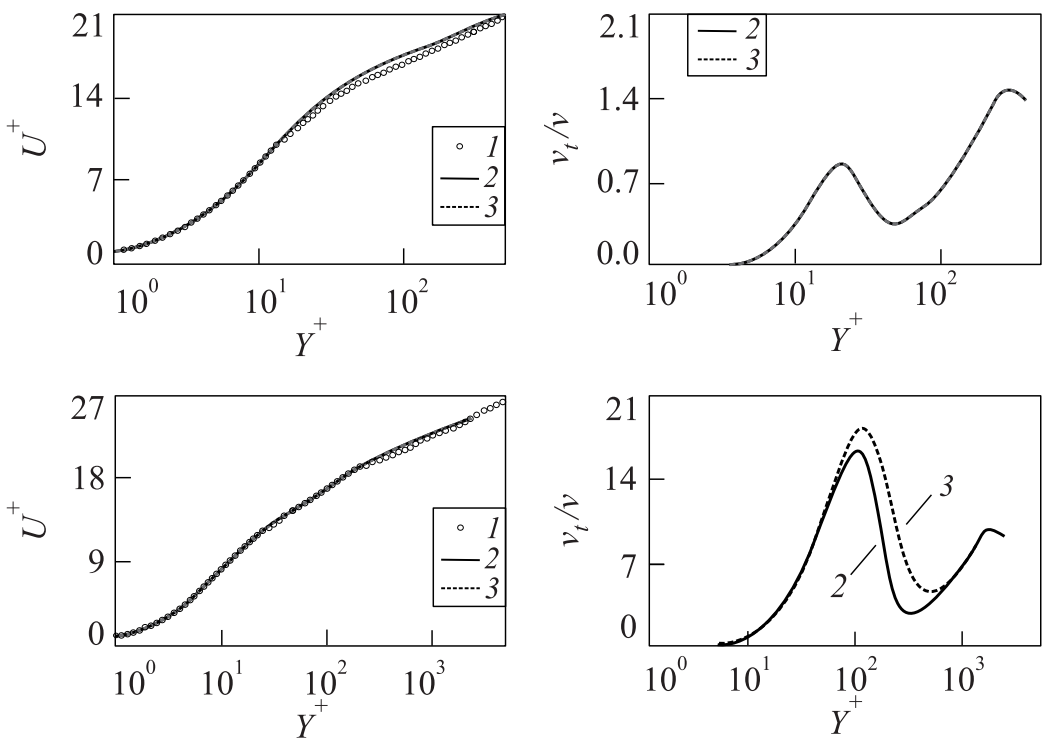

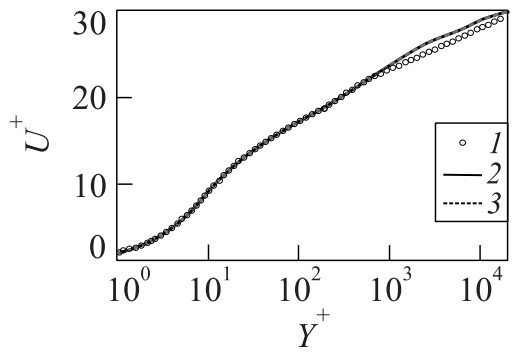

(a)

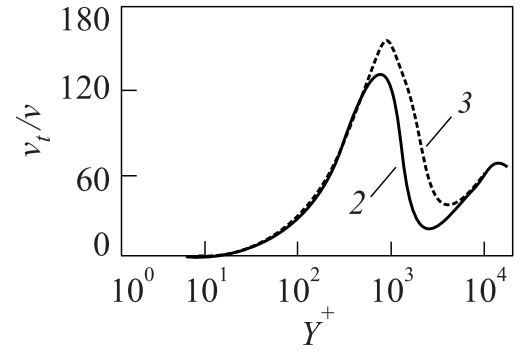

(b)

Figure 6 Effect of constant $C_{d t 1}$ on the SST IDDES of the developed channel flow: velocity $(a)$ and eddy viscosity $(b)$ profiles at different Reynolds numbers: upper row $\operatorname{Re}_{\tau}=395$; middle row - 2400; and lower row $-\operatorname{Re}_{\tau}=18,000 ; 1$ - correlation; 2 IDDES, $C_{d t 1}=8 ;$ and $3-$ IDDES, $C_{d t 1}=20$ 
correlation of Reichardt [16] are presented in Fig. 6 where the mean velocity and eddy viscosity profiles for different Reynolds numbers are depicted. It can be seen that the effect of changing $C_{d t 1}$ on these profiles is negligible which suggests that the new value of the constant does not cause any damage to the wall-modeling capability of the SST-based IDDES formulation.

Next, the performance of the model for the backward-facing step flow was investigated. As mentioned above, in this flow, both branches of IDDES, DDES, and WMLES are active: the model effectively performs as DDES in the attached flow region upstream of the step and in the attached boundary layer on the upper straight wall of the channel and as WMLES in the recirculation zone and downstream of the reattachment on the step-wall. The results of the SSTIDDES of the flow carried out with $C_{d t 1}=8$ and 20 are depicted in Fig. 7. The figure suggests that the variation of the constant does not affect the performance of the model and yields virtually identical results for all considered quantities. The only visible difference is seen in the skin friction coefficient, but it is also marginal. Other than that, the version of SST-IDDES with a new value of

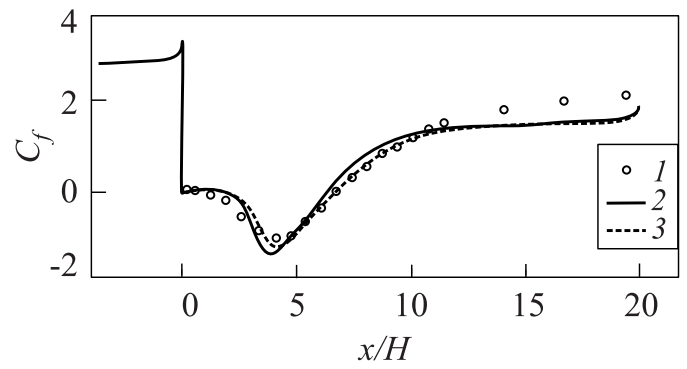

(a)

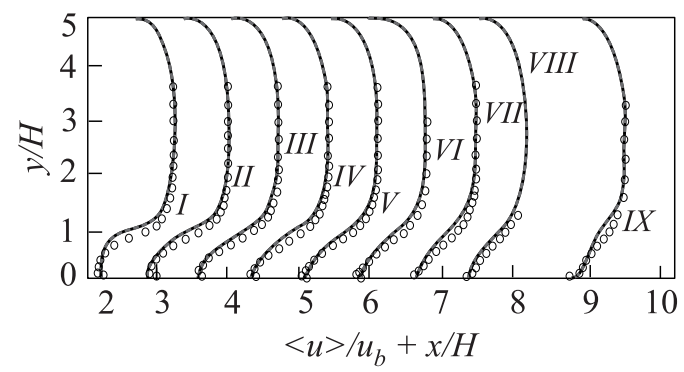

(b)

Figure 7 Effect of constant $C_{d t 1}$ on the SST IDDES of the BFS flow: $(a)$ skin friction coefficient distribution, and $(b)$ profiles of streamwise velocity $\langle u\rangle$ plotted at $x / H=2.2(I), 3.0(I I), 3.7(I I I), 4.5(I V), 5.2(V), 5.9(V I), 6.7$ (VII), 7.4 (VIII), and $8.7(I X) ; 1$ - experiment; 2 - IDDES, $C_{d t 1}=8 ; 3-$ IDDES, $C_{d t 1}=20$ 
$C_{d t 1}=20$ provides very good agreement with the experiment, thus supporting the credibility of the proposed modification of the model.

\section{SIMPLIFICATION OF SHEAR STRESS TRANSPORT BASED IDDES}

In addition to the delay function similar to that of DDES, the IDDES approach involves the elevating function $f_{e}$, aimed at preventing the excessive reduction
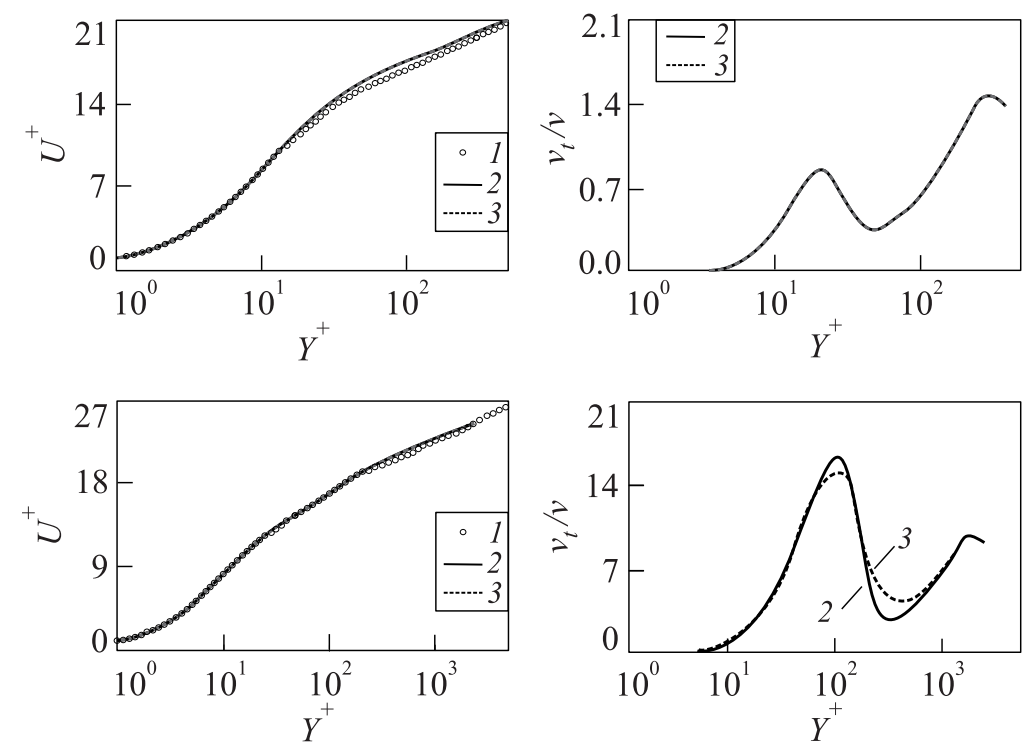

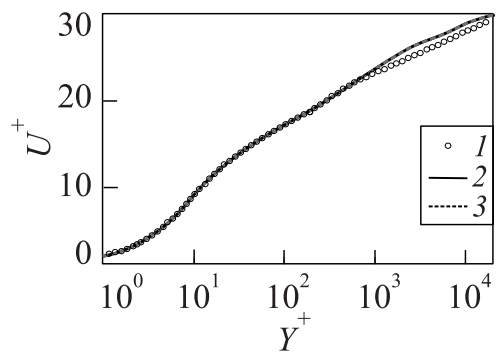

(a)

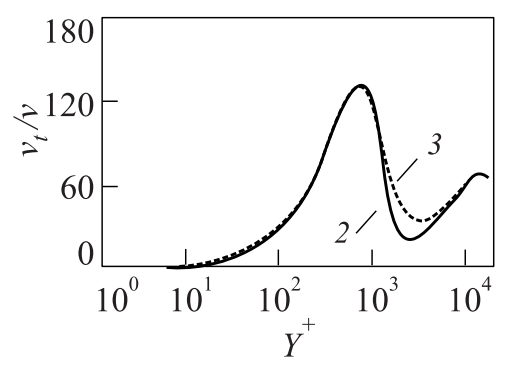

(b)

Figure 8 Comparison of mean velocity $(a)$ and eddy viscosity $(b)$ profiles and in developed channel flow predicted by full and simplified versions of the SST-based IDDES: upper row $-\operatorname{Re}_{\tau}=395$; middle row -2400 ; and lower row $-\operatorname{Re}_{\tau}=18,000 ; 1-$ correlation; 2 - IDDES; and 3 - simplified IDDES 


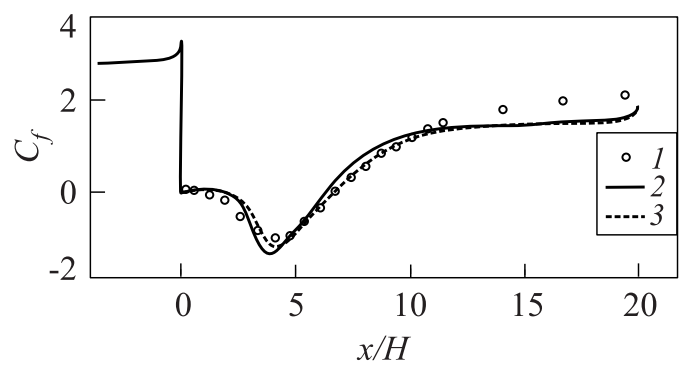

(a)

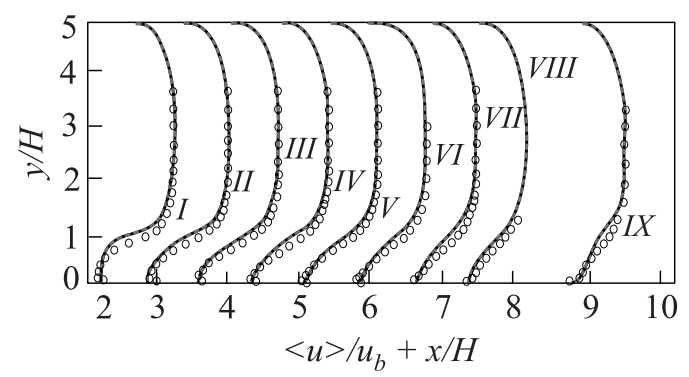

(b)

Figure 9 Comparison of skin friction coefficient distribution over the step-wall $(a)$ and profiles of streamwise velocity $\langle u\rangle(b)$ predicted by full and simplified versions of SSTIDDES in the BFS flow. The profiles are plotted at $x / H=2.2(I), 3.0(I I), 3.7(I I I)$, $4.5(I V), 5.2,(V) 5.9(V I), 6.7$ (VII), 7.4 (VIII), and 8.7 (IX); 1 - experiment; 2 IDDES; and 3 - simplified IDDES

of the RANS stresses typically observed in the vicinity of the RANS and LES interface and causing the so-called Log-Layer Mismatch (LLM) [4] in both DES and DDES when the models are applied to the attached flows. As shown in [4], within the SA-IDDES model, this function is more "aggressive" than within the SST-IDDES model, meaning it elevates the RANS model eddy-viscosity more strongly for the SA-IDDES model. Considering that it noticeably complicates the IDDES formulation and makes an analysis and understanding of the model performance nontrivial, it was tempting to evaluate the effect of removing $f_{e}$ from the SST-based formulation of IDDES, i. e., setting $f_{e}=0$. Hereafter, this model is referred to as simplified IDDES, in contrast to IDDES, which means SST-based IDDES with $C_{d t 1}=8$ considered in section 3 . The simplified IDDES has been evaluated based on a range of flows, with confined areas of attached and separated flow regions. The obtained results are presented below.

The simplified IDDES was first applied again to the channel flow where the effect of omitting $f_{e}$ had been expected to be most noticeable. The three flow 
regimes considered in subsection 4.1 were simulated with the use of the problem setup and boundary conditions described in subsection 4.1. The results of the simulations in the form of the mean velocity and eddy viscosity profiles are shown in Fig. 8. The figure suggests that, in line with the expectations, the simplified IDDES does cause somewhat stronger LLM at the low and moderate values of the Reynolds number, but the effect is marginal. Thus, as far as this flow is concerned, the simplification of the original formulation of the SST-based IDDES [4] is justified.

Some results of SST-based IDDES and simplified IDDES of the backwardfacing step flow (see subsection 3.2) are shown in Fig. 9. It suggests that both models perform practically identically, thus supporting the positive conclusion formulated regarding the simplified IDDES model based on the simulation of the developed channel flow presented in the previous section.

The results of the simulations for the periodic hills flow (see subsection 3.3) with the use of both original and simplified IDDES models are presented in Fig. 10. Just as in the two flows considered above (the plane channel and BFS),

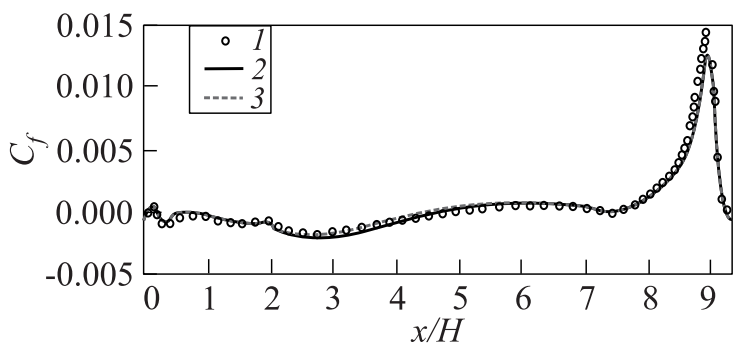

(a)

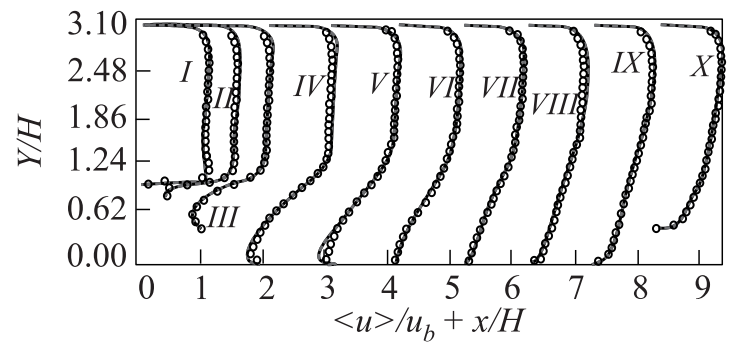

(b)

Figure 10 Comparison of skin friction distribution $(a)$ and streamwise velocity profiles $\langle u\rangle(b)$ predicted by full and simplified versions of the SST-based IDDS with LES data [12]. The profiles are plotted at $x / H=0.05(I), 0.5(I I), 1$ (III), $2(I V)$, $3(V), 4(V I), 5(V I I), 6(V I I I), 7(I X)$, and $8(X) ; 1$ - LES; 2 - IDDES; and 3 simplified IDDES 


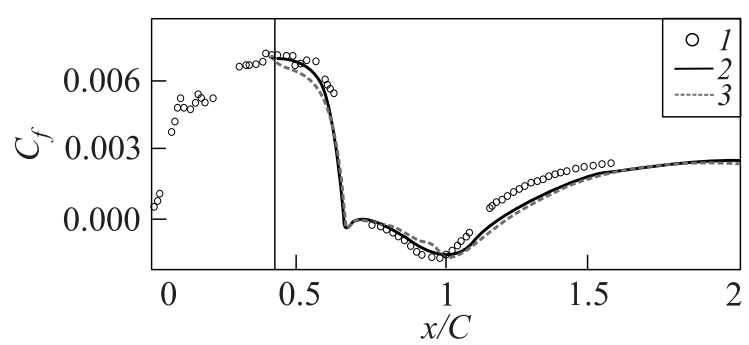

(a)

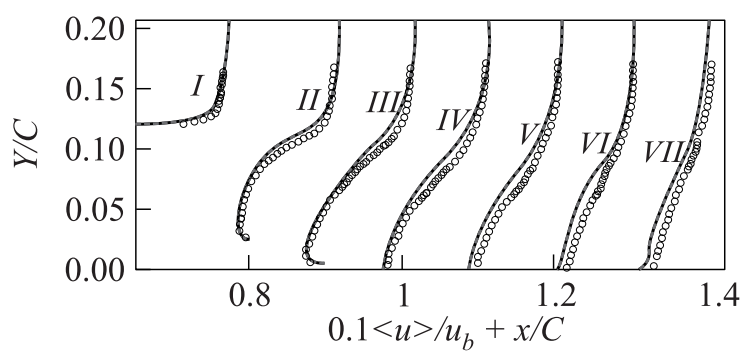

(b)

Figure 11 Comparison of skin friction coefficient distribution $(a)$ and profiles of streamwise velocity $\langle u\rangle(b)$ predicted by full and simplified versions of SST-based IDDES in the 2D wall-mounted hump flow with experimental data [10]. Profiles are plotted at $x / H=0.65(I), 0.8(I I), 0.9(I I I), 1.0(I V), 1.1(V), 1.2(V I)$, and 1.3 (VII); 1 - experiment; 2 - IDDES; and 3 - simplified IDDE

both models produce virtually identical predictions of the skin friction distribution (Fig. 10a) and for profiles of the mean streamwise velocity (Fig. 10b), which all very well agree with the reference LES solution of Breuer et al. [12].

Some typical results of the simulations of the $2 \mathrm{D}$ hump flow (see subsection 3.4) with the use of the full and simplified versions of the SST-based IDDES model are presented in Fig. 11. They show that similar to all the flows considered above, both versions yield close solutions. However, in this case, the difference between the two solutions is somewhat more pronounced. On the other hand, in terms of the agreement with the data, the full version, in general, does not surpass the simplified one and therefore, the simplification appears justified for this flow as well.

In Fig. 12, the results of the simulations of the hydrofoil with trailing edge separation flow (see subsection 3.5) with the use of full and simplified SST-based IDDES model versions are compared with each other. In addition, well-resolved LES $[17,18]$ and experimental data [13] are included. It can be seen that, again, virtually no differences are observed between the predictions of the full and 


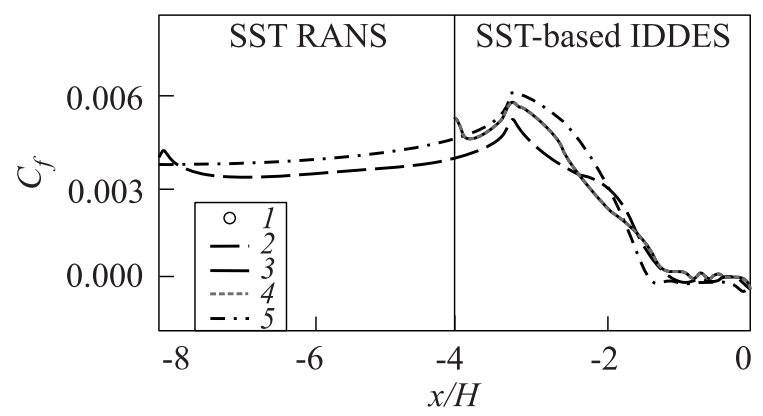

(a)

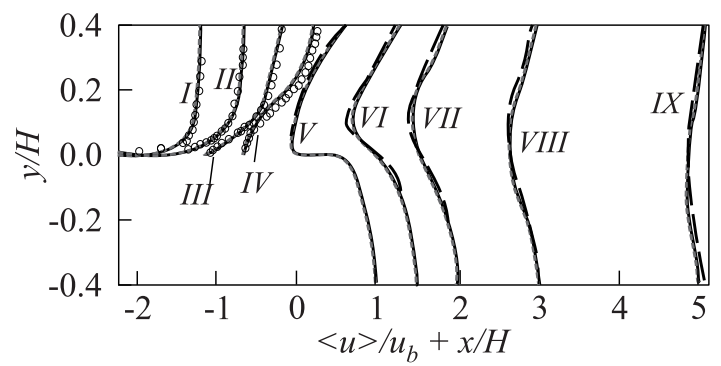

(b)

Figure 12 Comparison of skin friction coefficient distributions $(a)$ and profiles of streamwise velocity $\langle u\rangle(b)$ predicted by full and simplified versions of SST-based IDDES with similar LES results of [17, 18] and experimental data [13]. Profiles are plotted at $x / H=-2.125(I),-1.625(I I),-1.125(I I I),-0.625(I V), 0.0(V), 0.5(V I)$, $1.0(V I I), 2.0(V I I I)$, and $4.0(I X) ; 1$ - experiment; 2 - LES; 3 - IDDES; 4 simplified IDDES; and 5 - SST RANS

simplified versions of the SST-based IDDES models and that both agree fairly well with the full LES predictions and experimental data.

\section{CONCLUDING REMARKS}

Recalibration of the empiric constants $C_{d 1}$ and $C_{d 2}$ involved in the delay function $f_{d}$ of the SA-based DDES model was carried out in order to optimize the formulation when used with the SST-based DDES model. Simulations of different flows, both attached and separated, performed with the recalibrated constants have shown that they provide the same level of shielding for the SST-based DDES and IDDES variants from model stress depletion as achieved by the SA-IDDES models, on the one hand, and do not impair the turbulence resolving capability 
of the model in the separated flow regions, on the other hand. It has been shown also that the use of these constants within the SST-based IDDES model does not corrupt its WMLES capability in the attached flows.

In addition, a simplified version of SST-based IDDES is shown to perform virtually identical to its full version in all the considered flows suggesting that in the framework of the SST-based IDDES model this function is superfluous.

\section{ACKNOWLEDGMENTS}

The authors would like to acknowledge the financial support from the EU project ATAAC and from ANSYS Inc.

\section{REFERENCES}

1. Fröhlich, J., and D. Von Terzi. 2008. Hybrid RANS/LES methods for the simulation of turbulent flows. Progr. Aerospace Sci. 44:349-77.

2. Menter, F. R., M. Kuntz, and R. Langtry. 2003. Ten years of experience with the SST turbulence model. Turbulence Heat Mass Transfer 4:625-32.

3. Spalart, P. R., S. Deck, M. L. Shur, K. D. Squires, M. K. Strelets, and A. Travin. 2006. A new version of detached-eddy simulation, resistant to ambiguous grid densities. Theor. Comput. Fluid Dyn. 181-95.

4. Shur, M. L., P. R. Spalart, M. K. Strelets, and A. K. Travin. 2008. A hybrid RANSLES approach with delayed-DES and wall-modeled LES capabilities. Int. J. Heat Fluid Flow 29:1638-49.

5. ANSYS FLUENT 12.0 Theory Guide. 2009.

6. Rhie, C. M., and W. L. Chow. 1983. Numerical study of the turbulent flow past an airfoil with trailing edge separation. AIAA J. 21:1525-32.

7. Vandoormaal, J.P., and G.D. Raithby. 1984. Enhancements of the SIMPLE method for predicting incompressible fluid flows. Numer. Heat Transfer 7:147-63.

8. Vogel, J. C., and J. K. Eaton. 1985. Combined heat transfer and fluid dynamic measurements downstream of a backward-facing step. J. Heat Mass Transfer 107:92229.

9. Temmerman, L., and M. A. Leschziner. 2001. Large eddy simulation of separated flow in a streamwise periodic channel constriction. Symposium (International) Turbulent Shear Flow Phenomena. Stockholm, Sweden.

10. Greenblatt, D., K. B. Paschal, Y. Chung-Sheng, and J. Harris. 2005. A separation control CFD Validation Test Case Part 2. Zero efflux oscillatory blowing. AIAA Paper No. 2005-0485.

11. Adamian, D., and A. Travin. 2010. An efficient generator of synthetic turbulence at RANS-LES interface in embedded LES of wall-bounded and free shear flows. ICCFD 6 . 
12. Breuer, M., N. Peller, C. Rapp, and M. Manhart. 2009. Flow over periodic hills numerical and experimental study in a wide range of Reynolds numbers. Computers Fluids 38:433-57.

13. Blake, W.K. 1975. A statistical description of pressure and velocity fields the trailing edge of a flat strut. David Taylor Naval Ship R\&D Center Report 4241. Bethesda.

14. Travin, A., M. Shur, M. Strelets, and P. R. Spalart. 2002. Physical and numerical upgrades in the detached-eddy simulation of complex turbulent flows. In: Advances in LES of complex flows. 239-54.

15. Rumsey, C.L., T. B. Gatski, W.L. Sellers, V.N. Vatsa, and S. A. Viken. 2004. Summary of the 2004 CFD Validation Workshop on Synthetic Jets and Turbulent Separation Control. AIAA Paper No. 2004-2217.

16. Reichardt, H. 1951. Vollstandige darstellung der turbulenten geschwindigkeitsverteilung in glatten leitungen. Zeitschrift fur Angewandte Mathematik und Mechanik 31:208-19.

17. Wang, M., and P. Moin. 2000. Computation of trailing-edge flow and noise using large-eddy simulation. AIAA J. 38:2201-9.

18. Wang, M., and P. Moin. 2002. Dynamic wall modeling for large-eddy simulation of complex turbulent flows. Phys. Fluids 14:2043-51. 